\title{
Dexamethasone improved disability in acute bacterial meningitis
}

\author{
de Gans J,van de Beek D. Dexamethasone in adults with bacterial meningitis. N Engl J Med 2002;347:1549-56. \\ QUESTION: In patients with acute bacterial meningitis, does adjunctive treatment with \\ dexamethasone improve outcomes more than placebo?
}

\section{Design}

Randomised (allocation concealed*), blinded \{clinicians, patients, data collectors, outcome assessors, data analysts, data monitoring committee, and manuscript writers $\}$ †* placebo controlled trial with 8 weeks of follow up.

\section{Setting}

52 centres in the Netherlands, Belgium, Germany, Denmark, and Austria.

\section{Patients}

301 patients (mean age $45 \mathrm{y}, 56 \%$ men) who had suspected meningitis in combination with cloudy cerebrospinal fluid, bacteria in cerebrospinal fluid on Gram staining, or cerebrospinal fluid leukocyte count $>1000 / \mathrm{mm}^{3}$. Exclusion criteria included hypersensitivity to $\beta$ lactam antibiotics or corticosteroids; cerebrospinal shunt; antibiotics in the past 48 hours; and history of active tuberculosis or fungal infection, head trauma, neurosurgery, or peptic ulcer disease. All patients were included in the analysis.

\section{Intervention}

Patients were allocated to intravenous dexamethasone, $10 \mathrm{mg}$ every 6 hours for 4 days $(\mathrm{n}=157)$, or placebo $(\mathrm{n}=144)$. All patients received parenteral antibiotics.

\section{Main outcome measures}

The primary outcome was score on the Glasgow Outcome Scale (score of $5=$ favourable outcome [mild or no disability], and 1-4=unfavourable outcome [range, death to moderate disability]). Secondary outcomes were death, focal neurological abnormalities, hearing
Source of funding: NV Organon. DrJ de Gans,

University of

Amsterdam,

Amsterdam, The

Netherlands.

j.degans@amc.uva.nl

Abstract and

commentary also

Club.
For correspondence:

appear in ACP Journal loss, gastrointestinal bleeding, fungal infection, herpes zoster, and hyperglycaemia.

\section{Main results}

Analysis was by intention to treat. Fewer patients who received dexamethasone had an unfavourable outcome than did patients who received placebo (table). Also, fewer patients in the dexamethasone group died than in the placebo group (table). The groups did not differ for any other secondary outcomes. Dexamethasone and placebo groups did not differ for adverse effects.
Dexamethasone v placebo for bacterial meningitis at 8 weeks

\begin{tabular}{lclll} 
Outcomes & Dexamethasone & Placebo & RRR (95\% CI) & NNT (CI) \\
Unfavourable outcome & $15 \%$ & $25 \%$ & $41 \%(7$ to 63$)$ & 10 (5 to 73$)$ \\
\hline Death & $7 \%$ & $15 \%$ & $52 \%(5$ to 75$)$ & 13 (7 to 163$)$ \\
\hline
\end{tabular}

fUnfavourable outcome $=$ score of $1-4$ on the Glasgow Outcome Scale: death, vegetative state, severe disability, or moderate disability. Abbreviations defined in glossary; RRR, NNT, and Cl calculated from data in article.

\section{Conclusion}

In patients with acute bacterial meningitis, adjunctive treatment with dexamethasone was more effective than placebo in improving disability and reducing death.

*See glossary.

$\dagger$ Information provided by author.

\section{COMMENTARY}

Before the study of de Gans and van de Beek, only 3 randomised trials evaluated dexamethasone in adults with bacterial meningitis. The first, ${ }^{1}$ done in Egypt, showed a survival benefit in a subset of adults with pneumococcal meningitis. However, $60 \%$ of the patients were comatose at entry, and antibiotics were administered intramuscularly. The second study, ${ }^{2}$ which showed a trend to better outcomes with dexamethasone, enrolled only 60 patients of a planned 256, and dexamethasone could be administered up to 3 hours after the first dose of antibiotics. The third study, ${ }^{3}$ done in India, enrolled only 30 patients, and dexamethasone was started after antibiotics.

The study by de Gans and van de Beek addresses the limitations of the previous studies. The sample size was 301 , and dexamethasone was given before or at the same time as the first antibiotic dose. The benefit of dexamethasone was largely restricted to those with pneumococcal meningitis.

The study was done principally in the Netherlands, where strains of Streptococcus pmeumoniae with reduced susceptibility to penicillin are rare. In centres where reduced susceptibility of S. pneumoniae to a third generation cephalosporin exists, it is common for vancomycin to be used with a third generation cephalosporin for empirical treatment of bacterial meningitis pending culture and susceptibility results. However, evidence from animal studies shows that dexamethasone reduces penetration of vancomycin into infected cerebrospinal fluid. ${ }^{4}$ Thus, there is concern that use of dexamethasone with vancomycin could compromise the efficacy of vancomycin in third generation cephalosporin resistant strains. ${ }^{5}$ However, the vast majority of cases of bacterial meningitis are not caused by $S$. pneumoniae with reduced susceptibility to third generation cephalosporins. Thus, even if adjunctive dexamethasone was deleterious in patients with meningitis caused by S. pmeumoniae resistant to third generation cephalosporins (which has not been established), many more patients would benefit from dexamethasone. Dexamethasone should now be considered the standard of care, provided that it is initiated before or at the same time as antibiotics.

Stephen D Shafran, MD University of Alberta, Edmonton, Alberta, Canada

1 Girgis NI, Farid Z, Mikhail IA, et al. Dexamethasone treatment for bacterial meningitis in children and adults. Pediatr Infect Dis J 1989:8:848-51.

2 Thomas R, Le Tulzo Y, Bouget J, et al. Trial of dexamethasone treatment for severe bacterial meningitis in adults. Adult Meningitis Steroid Group. Intensive Care Med 1999;25:475-80.

3 Bhaumik S, Behari M. Role of dexamethasone as adjunctive therapy in acute bacterial meningitis in adults. Neurology India $1998 ; \mathbf{4 6}: 225-8$

4 Martínez-Lacasa J, Cabellos C, Martos A, et al. Experimental study of the efficacy of vancomycin, rifampicin and dexamethasone in the therapy of pneumococcal meningitis. IAntimicrob Chemother 2002;49:507-13.

5 Viladrich PF, Gudiol F, Liñares J, et al. Evaluation of vancomycin for therapy of adult pneumococcal meningitis. Antimicrob Agents Chemother 1991;35:2467-72. 